\title{
EFEITO DE Beauveria bassiana (Bals.) VUILLEMIN E Metarhizium anisopliae (Metsch.) SOROKIN NOS PARÂMETROS BIOLÓGICOS DE Trichogramma atopovirilia OATMAN \& PLATNER, 1983 (HYMENOPTERA: TRICHOGRAMMATIDAE)
}

\author{
Effect of Beauveria bassiana (Bals.) Vuillemin and Metarhizium anisopliae (Metsch.) Sorokin on \\ the biological parameters of Trichogramma atopovirilia Oatman \& Platner \\ (Hymenoptera: Trichogrammatidae)
}

\author{
Ricardo Antonio Polanczyk ${ }^{1}$, Dirceu Pratissoli ${ }^{2}$, Leandro Pin Dalvi ${ }^{3}$, \\ Eduardo Domingos Grecco ${ }^{4}$, Cláudio Roberto Franco ${ }^{5}$
}

\begin{abstract}
RESUMO
Dois experimentos foram realizados para avaliar o efeito de duas formulações comerciais à base de Beauveria bassiana (Bals.) Vuillemin e Metarhizium anisopliae (Metsch., Sorokin sobre os parâmetros biológicos de Trichogramma atopovirilia Oatman \& Platner, 1983. No primeiro experimento, cartelas com ovos de Spodoptera frugiperda, 1797 foram mergulhadas em suspensões preparadas com os produtos e, em seguida, colocadas à disposição dos parasitóides para oviposição, durante um período de 24 horas. No segundo, fêmeas do parasitóide foram alimentadas com uma solução mel e suspensão de conídios. Em ambos os experimentos as fêmeas mortas foram colocadas em uma câmara úmida para observar a esporulação dos fungos. Os tratamentos foram mantidos em câmara climatizada com temperatura de $25 \pm 1^{\circ} \mathrm{C}$, umidade relativa de $70 \pm 10 \%$ e fotofase de 14 horas. Avaliou-se a longevidade e mortalidade dos adultos, índice de parasitismo, emergência do parasitóide, número de indivíduos por ovo e razão sexual dos descendentes. As formulações não interferiram nos parâmetros avaliados e não foi observada a esporulação do fungo no cadáver do parasitóide adulto. É possível inferir que T. atopovirilia e os fungos entomopatogênicos B. bassiana e M. anisopliae são compatíveis e podem ser empregados simultaneamente em programas de manejo integrado de S. frugiperda.
\end{abstract}

Termos para indexação: Parasitóide, entomopatógeno, manejo integrado de pragas.

\begin{abstract}
Two bioassays were performed to evaluate the effect of two biopesticides based on Beauveria bassiana (Bals.) Vuillemin and Metarhizium anisopliae (Metsch.) Sorokin on the biological parameters of Trichogramma atopovirilia Oatman \& Platner, 1983. In the first one, displays with $S$. frugiperda, 1797 eggs were dropped into the biopesticide suspension and offered to the parasitoid females for 24 hours. In the second one, parasitoid females were fed with a suspension containing honey and biopesticide suspension. In both cases, after the parasitoid death they were mantained into a humid chamber to observe fungus sporulation. The experiments were maintained in a climatized chamber at $25 \pm 1^{\circ} \mathrm{C}, \mathrm{RH} 70 \pm 10 \%$, and photophase of 14 hours. The longevity and adult mortality, total parasitism, progeny emergency, number of individuals per egg and sexual ratio were analyzed. The biopesticides did not affect the evaluated parameters and no fungus sporulation was observed in dead females. It is possible to assert that the entomopathogenic fungi $B$. bassiana and $M$. anisopliae can be used with $T$. atopovirilia in IPM $S$. frugiperda systems.
\end{abstract}

Index terms: Parasitoid, entomopathogen, integrated pest management.

(Recebido em 9 de abril de 2008 e aprovado em 18 de agosto de 2009)

\section{INTRODUÇÃo}

A lagarta-do-cartucho, Spodoptera frugiperda (J.E. Smith) (Lepidoptera: Noctuidae), é considerada a principal praga do milho no Brasil. As lagartas se alimentam da planta em todas as fases de desenvolvimento e podem causar perdas de até $37 \%$ da produção. Em razão do seu hábito polífago, essa praga ocorre durante o ano todo, sendo que a sua incidência e severidade vêm, aumentando não só em áreas cultivadas com milho, mas também em outras culturas, como o algodão (Cruz et al., 1999; Gallo et al., 2002; Pinto et al., 2004).

O controle dessa praga tem sido realizado com agrotóxicos, geralmente de amplo espectro e de custo elevado, com altos riscos de intoxicações para o homem,

¿Universidade Estadual Paulista "Julho de Mesquita Filho"/UNESP - Via de Acesso Professor Paulo Donato Castellane - s/n - Jaboticabal, SP rapolanc@yahoo.com.br

2Universidade de São Paulo/USP - Escola Superior de Agricultura "Luiz de Queiroz"/ESALQ - Departamento de Produção Vegetal - Alegre, ES

${ }^{3}$ Universidade Federal de Viçosa - Departamento de Fitotecnia - Viçosa, MG

${ }^{4}$ Universidade Federal do Espírito Santo/UFES - Centro de Ciências Agrárias - Alegre, ES

5Universidade de São Paulo/USP - Escola Superior de Agricultura "Luiz de Queiroz"/ESALQ - Lages, SC 
outros animais e para os inimigos naturais, envolvendo parasitóides, predadores e entomopatógenos, além de problemas relacionados à resistência (Viana \& Prates, 2003). Portanto, torna-se importante o estudo de novas táticas de controle menos agressivas ao meio ambiente $\mathrm{e}$ que estejam de acordo com a filosofia básica do manejo integrado de pragas.

Como alternativa ao uso dos inseticidas convencionais, destacam-se os agentes de controle biológico, pela sua alta eficácia no controle de pragas, seletividade e baixo risco ao meio ambiente. Dentre os principais agentes de controle biológico, destacam-se os parasitóides de ovos do gênero Trichogramma (Hymenoptera: Trichogrammatidae) e os fungos entomopatogênicos, principalmente Beauveria bassiana (Bals.) (Vuill) e Metarhizium anisopliae (Metsch.) Sorok. (Alves, 1998; Parra et al., 2002; Thuler et al., 2008; Zago et al., 2008).

De acordo com Bezerra \& Parra (2004), Trichogramma atopovirilia Oatman \& Platner é a espécie que demonstra maior aceitação pelos ovos de $S$. frugiperda, a mais agressiva e com maior potencial para utilização no campo. Dentre os fungos entomopatogênicos, B. bassiana e $M$. anisopliae apresentam alta virulência contra uma ampla gama de insetos hospedeiros (Cruz, 1995; Alves, 1998) e potencial para o controle de $S$. frugiperda (Castanheira et al., 1993; Polanczyk \& Alves, 2005).

Parasitóides e entomopatógenos podem ser utilizados simultaneamente em programas de manejo integrado de pragas, sendo necessário verificar a compatibilidade entre eles. De acordo com Magalhães et al. (1998), as relações patógenos e parasitóides podem ser de diversos tipos, inclusive prejudiciais ao parasitóide e ao hospedeiro. As interações prejudiciais ao parasitóide podem ocorrer em razão da infecção direta, toxinas produzidas pelo microrganismo, morte antecipada do hospedeiro, redução na população do hospedeiro e alterações fisiológicas e nutricionais do hospedeiro.

Dela Rosa et al. (2001) ressaltam que B. bassiana e $M$. anisopliae já foram isolados de diversas espécies de microhimenópteros parasitóides, enfatizando a necessidade de avaliar os possíveis efeitos negativos dos fungos entomopatogênicos sobre estes inimigos naturais.

Neste trabalho, objetivou-se avaliar o efeito de duas formulações comerciais à base de B. bassiana e $M$. anisopliae aplicadas sobre ovos de $S$. frugiperda nos parâmetros biológicos de T. atopovirilia.

\section{MATERIAL E MÉTODOS}

Os métodos para a realização dos bioensaios descritos a seguir foram baseados nos princípios descritos por Consoli et al. (2001), Williams \& Price (2004) e Moura et al. (2006). Esses autores avaliaram o efeito de inseticidas, em condições de laboratório, sobre inimigos naturais.

A pesquisa foi conduzida no Laboratório de Entomologia e Fitopatologia do Centro de Ciências Agrárias da Universidade Federal do Espírito Santo, em câmara climatizada com temperatura de $25 \pm 1^{\circ} \mathrm{C}$, umidade relativa de $70 \pm 10 \%$ e fotofase de 14 horas. Para cada tratamento foram utilizadas 40 fêmeas recém emergidas de T. atopovirilia, que foram individualizadas em tubos de vidro $(8,0 \times 2,5 \mathrm{~cm})$ tamponados com filme plástico de PVC.

No primeiro experimento, cada fêmea (repetição) recebeu uma cartela $(4,0 \times 2,0 \mathrm{~cm})$ de cartolina azul celeste contendo 30 ovos do hospedeiro $S$. frugiperda com, no máximo, 12 horas de idade, colados sobre goma arábica a $10 \%$. Para a aplicação dos tratamentos foram preparadas suspensões com o bioinseticida Boveril WP PL63 (B. bassiana) e Metarril WP E9 (M. anisopliae) na concentração $10^{7}$ conídios $\mathrm{mL}^{-1}$. Essa concentração é discriminatória em testes de patogenicidade e serve para determinar se o entomopatógeno em questão tem ação sobre o organismo alvo ou não. Caso for positivo, procedese a estimativa da concentração letal média $\left(\mathrm{CL}_{50}\right)$ para estudar a agressividade do entomopatógeno para o organismo alvo, conforme Haddad (1998). As cartelas foram tratadas por imersão durante cinco segundos, nessa suspensão ou em água destilada (testemunha). Para a eliminação do excesso de água, as cartelas foram colocadas sobre papel de filtro, à temperatura ambiente. A fêmea permaneceu em contato com os ovos por um período de 24 horas, após o qual foi retirada. Essa fêmea foi morta e colocada em câmara úmida para observar a esporulação do fungo. Os parâmetros biológicos avaliados foram: capacidade de parasitismo, porcentagem de emergência do parasitóide (viabilidade), número de indivíduos por ovo e razão sexual.

No segundo experimento, para avaliação da longevidade do adulto, repetiu-se o procedimento adotado na avaliação dos demais parâmetros biológicos, porém foi pulverizada uma suspensão com o bioinseticida Boveril WP PL63 (B. bassiana) ou Metarril WP E9 (M. anisopliae), na mesma concentração e dosagens do teste anterior, na parte interna dos tubos. Após a evaporação do excesso de água, fêmeas do parasitóide foram colocadas no interior dos tubos para entrar em contato com o fungo. $\mathrm{Na}$ testemunha, foi pulverizada apenas água destilada no interior dos tubos de vidro. A fêmea foi mantida no interior do tubo até a sua morte, quando foi transferida para câmara úmida para a observação da esporulação. 
O delineamento experimental foi inteiramente casualizado e as médias foram comparadas pelo teste de Tukey a 5\% com o programa SAEG Sistema para Análises Estatísticas, versão 9.1.

\section{RESULTADOS E DISCUSSÃO}

A longevidade do parasitóide adulto não foi afetada pelos tratamentos (Tabela 1) e não foi observada mortalidade do parasitóide adulto, pela ingestão do fungo ou pelo contato do parasitóide com fungo através do ovipositor. Os resultados obtidos por (Dalvi et al., 2007) corroboram com os dados obtidos neste trabalho, pois os autores verificaram a seletividade do fungo entomopatogênico Lecanicillium lecanii para $T$. atopovirilia, sendo avaliados naquele caso, os mesmos parâmetros avaliados no presente estudo. A seletividade dos fungos entomopatogênicos para os inimigos naturais foi ressaltada por Alves (1998) como extremamente vantajosa em relação aos agrotóxicos convencionais.

A capacidade de parasitismo de T. atopovirilia não diferiu significativamente entre os tratamentos (Tabela 1). Assim, tanto ovos tratados com os patógenos, como os não tratados, foram visitados e parasitados pelas fêmeas do microhimenóptero. O índice de parasitismo dos ovos tratados com os patógenos $(68,9 \%)$ foi próximo ao encontrado por Bezerra \& Parra (2004) para T. atopovirilia $(63,2 \%)$ quando avaliaram o desempenho desse parasitóide sobre ovos de $S$. frugiperda.

Não se observou diferença na viabilidade de $T$. atopovirilia entre a testemunha e os tratamentos, sendo esta superior a 95\% para ambos os fungos (Tabela 1). Essa viabilidade é bem próxima ao valor de $96 \%$ encontrado por Nicoli et al. (2004), quando avaliaram esse parâmetro com T. atopovirilia sobre ovos de Anagasta kuehniella (Zeller). Segundo Navarro (1998), a viabilidade ideal para o controle da qualidade de Trichogramma deve ser acima de $85 \%$. Portanto, a presença dos fungos sobre os ovos do hospedeiro $S$. frugiperda não afetou sua qualidade, permitindo o desenvolvimento normal do parasitóide.
Quanto ao número de adultos emergidos por ovo, também não houve diferença significativa entre os tratamentos (Tabela 1). Os resultados diferem dos apresentados por Bezerra \& Parra (2004) que registraram 1,4 indivíduos por ovo de $S$. frugiperda. Uma das explicações para este fato pode ser a existência de variações entre linhagens de Trichogramma procedentes de diferentes localidades, as quais apresentam características bioecológicas particulares (Bleicher \& Parra, 1989).

Também, a razão sexual não apresentou diferença significativa entre os tratamentos, demonstrando que os fungos entomopatogênicos não tiveram influência sobre esse parâmetro biológico. Em todos os tratamentos, cerca de $80 \%$ dos indivíduos eram fêmeas. Esses resultados diferem dos apresentados por Navarro \& Marcano (1999) para T. atopovirilia em ovos de Helicoverpa zea, onde $56 \%$ dos indivíduos eram fêmeas. O parasitóide apresentou razão sexual satisfatória, pois os valores se situaram acima de 0,5 , valor mínimo exigido no controle de qualidade de Trichogramma (Navarro, 1998).

Os altos valores de razão sexual mostram a boa adequação dos ovos do hospedeiro $S$. frugiperda ao parasitismo, embora também possa ser decorrente do tempo de parasitismo de 24 horas, que corresponde à fase inicial da vida. De acordo com Hoffmann et al. (1995), a razão sexual dos descendentes é inversamente proporcional ao tempo de vida da fêmea.

Embora não tenha sido avaliado o efeito ovicida dos bioinseticidas, pois a duração da fase embrionária é de apenas três dias e o tempo inferior ao necessário para a avaliação da patogenicidade dos fungos, a ausência de efeitos dos tratamentos sobre os parâmetros biológicos do parasitóide e a sua progênie indica que ovos de $S$. frugiperda tratados com ambos os fungos não comprometeu o desempenho do parasitóide. Segundo Magalhães et al. (1998), em algumas situações o parasitóide é capaz de reconhecer o hospedeiro infectado por um patógeno pela coloração, forma, odor ou mudança de comportamento do hospedeiro infectado. Esses autores

Tabela 1 - Taxa de longevidade, parasitismo, viabilidade, indivíduos por ovo e razão sexual de T. atopovirilia sobre ovos de $S$. frugiperda não tratados e tratados com B. bassiana ou $M$. anisopliae. Temp.: $25 \pm 1^{\circ} \mathrm{C}, \mathrm{UR}=70 \pm 10 \%$, fotofase $=14 \mathrm{~h}$

\begin{tabular}{lccccc}
\hline Tratamento & Longevidade (dias) & Parasitismo (\%) & Viabilidade (\%) & Indivíduos/ovo & Razão sexual \\
\hline Testemunha & $8,00 \pm 0,47 \mathrm{a}$ & $71,29 \pm 1,23 \mathrm{a}$ & $95,30 \pm 0,95 \mathrm{a}$ & $1,06 \pm 0,16 \mathrm{a}$ & $0,81 \pm 0,09 \mathrm{a}$ \\
B. bassiana & $7,58 \pm 0,43 \mathrm{a}$ & $68,94 \pm 1,72 \mathrm{a}$ & $96,92 \pm 0,86 \mathrm{a}$ & $1,08 \pm 0,24 \mathrm{a}$ & $0,83 \pm 0,07 \mathrm{a}$ \\
M. anisopliae & $8,11 \pm 0,43 \mathrm{a}$ & $70,92 \pm 1,57 \mathrm{a}$ & $95,24 \pm 0,98 \mathrm{a}$ & $1,08 \pm 0,31 \mathrm{a}$ & $0,82 \pm 0,05 \mathrm{a}$ \\
\hline
\end{tabular}

Médias seguidas pela mesma letra na coluna não diferem pelo teste de Tukey $(p<0,05) . n=40$. 
ressaltaram ainda que o patógeno pode infectar diretamente o parasitóide, pois estes podem ser suscetíveis às doenças causadas por fungos que colonizam seus hospedeiros.

O fato do parasitóide não detectar e presença dos patógenos nos ovos e não ter sido afetado por estes, é extremamente importante, pois em uma situação de campo na qual seriam pulverizados bioinseticidas à base de $M$. anisopliae e B. bassiana e feita a liberação massal de $T$. atopovirilia, os bioinseticidas controlariam as lagartas de $S$. frugiperda e o parasitóide evitaria a eclosão de novas lagartas, sem problemas de incompatibilidade entre os agentes de controle. Com base nos resultados obtidos, pode-se inferir que o parasitóide $T$. atopovirilia e as formulações à base de B. bassiana (Boveril WP PL63) e M. anisopliae (Metarril WP E9) são compatíveis e podem ser empregados simultaneamente em programas de manejo integrado.

\section{CONCLUSÕES}

Os fungos entomopatogênicos Beauveria bassiana e Metarhizium anisopliae não tem efeito negativo sobre os parâmetros biológicos de Trichogramma atopovirilia o que possibilita o uso integrado desses agentes de controle biológico em programas de Manejo Integrado de Pragas.

\section{REFERÊNCIAS BIBLIOGRÁFICAS}

ALVES, S.B. Controle microbiano de insetos. In: Fungos entomopatogênicos. Piracicaba: FEALQ, 1998. p.289-382.

BEZERRA, E.B.; PARRA, J.R.P. Biologia e parasitismo de Trichogramma atopovirilia Oatman \& Platner e Trichogramma pretiosum Riley (Hymenoptera, Trichogrammatidae) em ovos de Spodoptera frugiperda (J.E. Smith) (Lepidoptera, Noctuidae). Revista Brasileira de Entomologia, Curitiba, v.48, n.1, p.119-126, 2004.

BLEICHER, E.; PARRA, J.R.P. Espécies de Trichogramma parasitóides de Alabama argillacea: I., biologia de três populações. Pesquisa Agropecuária Brasileira, Brasília, v.24, n.7, p.929-940, 1989.

CASTANHEIRA, R.S.; MUNHOZ, E.R.B.; COUTO, G.P. Eficiência da adubação do milho (Zea mays L.) sobre a eficiência de Beauveria bassiana (Bals.) Vuill., no controle da Spodoptera frugiperda (J.E. Smith, 1797). Ecossistema, Espírito Santo do Pinhal, v.18, n.2, p.119129, 1993.
CONSOLI, F.L.; BOTELHO, P.S.M.; PARRA, J.R.P.

Selectivity of insecticides to the egg parasitoid Trichogramma galloi Zucchi, 1988, (Hym., Trichogrammatidae). Journal of Applied Entomology, Hamburg, v.125, n.1, p.37-43, 2001.

CRUZ, I. A lagarta-do-cartucho na cultura do milho. Sete Lagoas: Embrapa-CNPMS, 1995. 45p. (Circular técnica, 21).

CRUZ, I.; FIGUEIREDO, M.L.C.; MATOSO, M.J. Controle biológico de Spodoptera frugiperda utilizando o parasitóide de ovos Trichogramma. Sete Lagoas: Embrapa-CNPMS, 1999. 40p. (Circular técnica, 30).

DALVI, L.P.; POLANCZYK, R.A.; PRATISSOLI, D.; MELO, R.L.de; HOLTZ, A.M. Seletividade de Lecanicillium lecanii (Zimm) Zare \& w. Gams (claseforma: Hyfhomycetes) ao parasitoide Trichogramma atopovirilia Oatman \& Platner, 1983 (Himenoptera: Trichogrammatidae). Ciência e Agrotecnologia, Lavras, v. 31, n. 5, p. 1392-1395, set,/out., 2007.

DE LA ROSA, W.; SEGURA, H.R.; BARRERA, J.F.; WILLIAMS, T. Laboratory evaluation of the impact of entomopathogenic fungi on Prorops nasuta (Hymenoptera: Bethylidae), a parasitoid of the coffee berry borer. Environmental Entomology, Lanham, v.29, n.1, p.126-131, 2001.

GALLO, D.; HAKANO O.; SILVEIRA NETO, S.; CARVALHO, R.P.L.; BAPTISTA, G.C. de; BERTI FILHO, E.; PARRA, J.R.P.; ZUCCHI, R.A.; ALVES, S.B.; VENDRAMIM, J.D.; MARCHINI, L.C.; LOPES, J.R.S.; OMOTO, C. Entomologia agrícola. Piracicaba: FEALQ, 2002. 920p.

HADDAD, M.L. Controle microbiano de insetos. In: ALVES, S.B. (Ed.). Utilização do Polo-PC para análise de Probit. Piracicaba: FEALQ, 1998. p.999-1014.

HOFFMANN, M.P.; WALKER, D.L.; SHELTON, A.M. Biology of Trichogramma ostriniae (Hym.:

Trichogrammatidae) reared on Ostrinia nubilalis (Lep.: Pyralidade) and survey for additional hosts.

Entomophaga, Paris, v.40, n.2, p.387-402, 1995.

MAGALHÃES, B.P.; MONNERAT, R.; ALVES, S.B. Controle microbiano de insetos. In: ALVES, S.B. (Ed.). Interações entre entomopatógenos, parasitóides e predadores. Piracicaba: FEALQ, 1998. p.195-216. 
MOURA, A.P.; CARVALHO, G.A.; PEREIRA, A.E.; ROCHA, L.C.D. Selectivety evaluation of insecticides used to control tomato pests to Trichogramma pretiosum. BioControl, Dordrecht, v.51, n.6, p.769-778, 2006.

NAVARRO, M.A. Trichogramma spp. procucción, uso y manejo en Colombia. Guadalajara de Buga: Imprectec, 1998. 176p.

NAVARRO, R.; MARCANO, R. Preferencia de Trichogramma pretiosum Riley y T. atopovirilia Oatman y Platner por huevos de Helicoverpa zea (Boddie) de diferentes idades. Boletim de Entomologia Venezuelana, Maracay, v.14, n.1, p.87-93, 1999.

NICOLI, E.M.; PRATISSOLI, D.; REIS, E.F.; SANTOS, H.S. Viabilidade e razão sexual de Trichogramma atopovirilia Oatman \& Platner, 1983 (Hymenoptera, Trichogrammatidae) sob influência do hospedeiro Anagasta kuehniella (Zeller, 1879) (Lepidoptera, Pyralidae) em condições de laboratório. Entomologia Vecter, Rio de Janeiro, v.11, n.6, p.521-533, 2004.

PARRA, J.R.P.; BOTELHO, P.S.M.; CORRÊAFERREIRA, B.S.; BENTO, J.M.S. Controle biológico no Brasil: parasitóides e predadores. São Paulo: Manole, 2002. 635p.

PINTO, A.S.; PARRA, J.R.P.; OLIVEIRA, H.N. de. Pragas e insetos benéficos do milho e sorgo: guia ilustrado. Ribeirão Preto: A.S. Pinto, 2004. 108p.
POLANCZYK, R.A.; ALVES, S.B. Interação entre Bacillus thuringiensis e outros entomopatógenos no controle de Spodoptera frugiperda. Manejo Integrado del Plagas Agroecologia, Turrialba, v.74, n.1, p.24-33, 2005.

THULER, R.T.; BORTOLI, S.A.de; GOULART, R.M.; VIANA, C.L.T.P.; PRATISSOLI. D. Interação tritrófica e influência de produtos químicos e vegetais no complexo: brássicas x traça-das-crucíferas x parasitóides de ovos. Ciência e Agrotecnologia, Lavras, v. 32, n. 4, p. 11541160, jul./ago., 2008.

VIANA, P.A.; PRATES, H.T. Desenvolvimento e mortalidade larval de Spodoptera frugiperda em folhas de milho tratadas com extrato aquoso de folhas de Azadirachta indica. Bragantia, Campinas, v.62, n.1, p.69-74, 2003.

WILLIAMS, L.; PRICE, L.D. A space-efficient contact toxicity bioassay for minute Hymenoptera, use to teste the effects of novel and conventional insecticides on the egg parasitoids Anaphes iole and Trichogramma pretiosum. BioControl, Dordrecht, v.49, n.2, p.163-185, 2004.

ZAGO, H.B.; PRATISSOLI, D.; BARROS, R.; GONDIM JÚNIOR, M.G.C. Tabela de vida de fertilidade de Trichogramma pratissolii Querino\& Zucchi, 2003 (Hymenoptera: Trichogrammatidae) em hospedeiros Alternativos, sob diferentes temperaturas. Ciência e Agrotecnologia, Lavras, v. 32, n. 4, p. 1214-1217, jul./ ago., 2008. 